Instituto Internacional de Investigación y Desarrollo Tecnológico Educativo INDTEC, C.A.

DOI: https://doi.org/10.29394/Scientific.issn.2542-2987.2018.3.7.5.99-120

OAI-PMH: http://www.indteca.com/ojs/index.php/Revista Scientific/oai

\title{
Aproximación Teórica para el uso de los Entornos Virtuales en el Proceso de Aprendizaje de los Estudiantes Universitarios
}

\author{
Autores: Elide del Rosario Castellanos Santiago \\ Universidad Nacional Experimental "Rafael María Baralt", UNERMB \\ elidecastellanos255@hotmail.com \\ Trujillo, Venezuela \\ Javier José Castro Capitillo \\ Universidad Nacional Experimental "Rafael María Baralt”, UNERMB \\ jccapitillo@hotmail.com \\ Trujillo, Venezuela
}

\section{Resumen}

La actual revisión teórica sirve para determinar el uso de los entornos virtuales en el proceso de aprendizaje de los estudiantes universitarios del Instituto, Universitario Tecnológico Mario Briceño Iragorri (IUTEMBI), en el Estado Trujillo. Los aportes teóricos que dieron soporte a la revisión teórica de acuerdo con las teorías de aprendizaje que soportan la indagación son los de Giroux, (1990), la Pedagogía Crítica o Sociocrítica. De La Torre, (2007), con el Constructivismo y Siemens, (2008), con el conectivismo como teoría del aprendizaje para la era digital. Además, está sustentada metodológicamente en una revisión documental. Las conclusiones, aportaron una reflexión que sirvió de andamio transformador donde el docente pueda cambiar y modificar su praxis pedagógica porque en este momento se exige una labor educativa tecnológicamente modernizada, con diferentes enfoques, de una manera integradora desde los contenidos, áreas y disciplinas que contempla el diseño curricular del sistema de educación universitaria. 


\title{
Theoretical Approach to the use of Virtual Environments in the Learning Process of University Students
}

\begin{abstract}
The current theoretical review serves to determine the use of virtual environments in the learning process of university students of the Institute, Technological University Mario Briceño Iragorri (IUTEMBI), in the Trujillo State. The theoretical contributions that supported the theoretical revision in accordance with the learning theories that support the inquiry are those of Giroux, (1990), Critical or Sociocritical Pedagogy. De La Torre, (2007), with Constructivism and Siemens, (2008), with connectivism as a theory of learning for the digital era. In addition, it is supported methodologically in a documentary review. The conclusions provided a reflection that served as a scaffolding transformer where the teacher can change and modify their pedagogical practice because at this time a technologically modernized educational work is required, with different approaches, in an integrating way from the contents, areas and disciplines that it contemplates. the curricular design of the university education system.
\end{abstract}

Keywords: information technology; learning process; students.

Date Received: 25-05-2017

Date Acceptance: 20-11-2017 


\section{Introducción}

La sociedad vigente está traspasando por un período de historicidad, es decir viviendo su transformación social, siguiendo un compás apresurado con cambios inmediatos que marcan en determinado momento. Asimismo, querer construir nuevas influencias orientadas al futuro, ante la mirada de distintas tendencias, es ofrecer aportes significativos al campo educativo donde se observa la dificultad de adecuación a los cambios sociales, tecnológicos, económicos y culturales en el acontecer de la colectividad. Según, Chiachio, Pievi, Echaverry y Gómez (2009), son "aspectos que hacen de las universidades un ámbito complicado, a veces conflictivo, inadecuado -en muchos aspectos- $y$ en parte ausente de entusiasmo, con situaciones carentes de apariencias y en búsquedas de nuevos sentidos" (pág. 201).

Dicho de otro modo, esto infiere un escenario actual donde la tecnologización provoca transformaciones en cuanto a la manera de concebir el conocimiento, constituir equipos de alto desempeño, comunicarse con los demás, originar nuevas maneras de aprendizaje, ocupar tiempo en adquirir habilidades tecnológicas. Es decir, donde el conocimiento aparece como el instrumento ineludible para la inclusión efectiva en el entorno social, condición que desafía a los sistemas educativos.

De hecho, en las observaciones realizadas por la autora se observó la baja calidad del proceso de aprendizaje referido a la estructura del entorno y la tecnología elegida. Además, la introducción de la Tecnología de la inferencia primordial tratar de cambiar las formas tradicionales de enseñanza. Aunado a esto, los docentes poseen escasamente aptitudes en materia de TIC por esto, no pueden enseñar de manera eficaz las asignaturas, porque no integran en su enseñanza concepciones, modelos y prácticas tecnológicas.

Por ende, con esta revisión teórica se pretende mostrar el impacto que las recientes tecnologías tienen en la innovación social. Sin duda, esto permea ámbitos de familia, educativo, laboral, comunitario, político y económico. En 
ese sentido, el contexto universitario debe adecuar las habilidades tecnologías a los procesos de aprendizaje, para determinar si los estudiantes poseen los conocimientos necesarios para su orientada utilización en el ámbito al que pertenecen. Así, esta indagación plantea como propósito general: Determinar el uso de los entornos virtuales en el proceso de aprendizaje de los estudiantes universitarios del Instituto, Universitario Tecnológico Mario Briceño Iragorri (IUTEMBI), en el Estado Trujillo.

\section{Proceso de Aprendizaje}

En este momento las tecnologías digitales admiten una mejor fluidez en las comunicaciones entre las personas, fragmentando barreras, ubicadas en espacio y tiempo, lo que ocasiona que el ser humano establezca mejores interacciones interpersonales. Destacando lo aludido, por Área, (2009a), "esta comunicación puede ser sincrónica, es decir, simultánea en el tiempo o asincrónica, el mensaje se emite y recibe en un período de tiempo posterior al emitido" (pág. 7). En otras palabras, comunicarse es hacerlo en el presente con las tecnologías a nuestro alcance tal como refiere el autor en recibirla al tiempo que se emite o esperar su llegada posteriormente a su emisión.

En consecuencia, ubicándonos en la temática mostrada los actuales retos educativos consisten en formar e instruir a los sujetos beneficiarios sobre aspectos donde sean capaces de distinguir lo significativo y dejar a un lado lo prolijo Al respecto, Área, (2009b), establece "las instituciones educativas se caracterizan por la lenta introducción de los cambios dentro de sus estructuras. En el contexto de las sociedades de la información, esta peculiaridad constituye una auténtica inadaptación a las necesidades formativas y a las exigencias organizacionales debido a un entorno en continuo movimiento y transformación" (pág. 10).

Parafraseando al autor, el auge tecnológico anhelado en las instituciones educativas se hace esperar, los escenarios están en ciertos 
contextos en espera de darles la utilidad que exigen estos tiempos posmodernos pero debido a ciertas debilidades y en muchas ocasiones a la falta de personal capacitado éstos no son utilizados como es el deber ser.

\subsection{Acción Docente en Entornos Virtuales.}

En este momento, la acción del docente universitario se entiende como la gestión para facilitar el aprendizaje donde las instituciones tituladas prosperan hacia la complementariedad del acostumbrado entorno educativo, en un reciente contexto que llamamos entorno virtual de aprendizaje (EVA). Actualmente, según Mestre, Fonseca, y Valdez, (2007):

Los géneros formativos dirigidos a personas adultas y en pleno ejercicio de su actividad profesional contemplan ya la complementariedad con el uso más o menos intensivo de las tecnologías aplicadas a la educación, ya sea a través de los recursos de la red Internet, de materiales multimedia de aprendizaje o de espacios relacionales virtuales de aprendizaje (pág. 34).

Es decir, con la anexión del uso intenso de las TIC en la educación, no podemos usar iguales dinámicos o metodologías docentes en el marco síncrono-presencial que en el asíncrono-virtual. La fuerte penetración tecnológica en los procesos educativos debe ser tratada para el establecimiento de estrategias concretas a favor del nuevo aprendizaje virtual o no presencial que hoy invade nuestros entornos académicos.

Aunado a esto, el estudiante universitario debe tener aptitudes para manejar lo relacionado con redes sociales, internet y entornos virtuales, lo que conlleva a sustentar las maneras de aprendizaje, dado que deben poseerse competencias en este caso tecnológicas para enseñar cómo usar estos medios, ofreciendo herramientas y recursos al alcance del aprendiz para que luego pueda aplicarlas de forma correcta en el contexto en el que se desenvuelve. 
Sin lugar a duda, los retos más significativos que actualmente tiene planeada la universidad, es lograr un mayor enlace entre la formación y el desarrollo profesional del estudiante. Sumando a esto, la universidad actual, sumergida en la globalización y las comunicaciones, debe favorecer el objetivo social que tiene encomendada, es decir la alineación y socialización de los jóvenes que constituirán parte en un futuro adyacente de la población activa que ha de llevar las riendas socialmente actuales.

De acuerdo con lo expuesto, el contexto universitario debe afrontar en este momento, la demanda de nuevas aptitudes no solo laboral sino en su vida. En conclusión, el avance de las tecnologías se torna fundamental en este estudio. No obstante, estas pueden desplegase ante una formación del profesorado que puede ser o no competente en esta área.

\subsection{Proceso de Aprendizaje: Trabajo Colaborativo en Entornos Virtuales.}

Hablar de Aprendizaje Colaborativo, en tiempos de posmodernidad ocurre en busca del mejoramiento en la educación, que permitan desplegar habilidades de ideología. No obstante, el ser humano nació para vivir en sociedad, su desarrollo espiritual, profesional es alcanzado en plenitud al interaccionar con otros. Así, debido a la dimensión individual de análisis, conceptualización, retención, siendo éste desarrollado a través del aprendizaje en colaboración con otros.

Asimismo, la representación del término aprendizaje colaborativo, es referente de métodos donde se desarrolla ampliamente el trabajo en equipo donde los grupos se hacen cooperadores para lograr un objetivo en común. Lo significativo es que se pudiera lograr este tipo de aprendizaje en los estudiantes del Instituto, Universitario Tecnológico Mario Briceño Iragorri, en el Estado Trujillo al dar uso a las TIC en su proceso de instrucción, mediante el uso del Internet incentivando la cooperación para la posterior formación de comunidades de aprendizaje virtual. 
De acuerdo con la temática, a juicio de Gosden, (1994), citado en Scagnoli, (2005a), "el aprendizaje colaborativo está inmerso en la teoría de constructivismo social y se centra en el proceso de construcción del conocimiento a través del aprendizaje que resulta de la interacción con un grupo y mediante tareas realizadas en cooperación con otros" (pág. 2). Lo expuesto por el autor, deja entrever lo importante de este tipo de aprendizaje para ser implementado en las aulas y así hacerlo de una forma constructivista donde el estudiante tenga oportunidades de aprender haciendo. A continuación, se presentan estos elementos:

1. Interdependencia positiva: Aplicado este elemento se hará que el estudiante sea responsable y capaz de compartir en equipo principios pues se tiene que el ser posee dependencia social y así mismo.

2. Promoción a la interacción: En esto se hará hincapié al trabajo colaborativo para que el estudiante interactúe para que los intereses sean compartidos y así crear conocimientos reales referidos al uso de los entornos virtuales en el proceso de aprendizaje, en este caso los estudiantes universitarios del Instituto, Universitario Tecnológico Mario Briceño Iragorri, en el Estado Trujillo.

3. Responsabilidad individual: Además, del trabajo en equipo la individualidad debe estar presente pues se tomará como aporte los conocimientos previos que posea cada estudiante para luego fusionarlos con los grupales y así unificar criterios.

4. Habilidades y destrezas de trabajo grupales: El aprender haciendo, significa construir colectivamente los distintos aprendizajes lo cual se espera lograr en el estudio mostrado.

5. Interacción positiva: el estudiante en su acontecer académico debe propiciar la interacción con los medios tecnológicos a su alcance de una forma auténtica tomando en cuenta que el propósito del aprendizaje colaborativo es construir conocimiento mediante la indagación, discusión y colaboración con 
los demás Scagnoli, (2005b, págs. 31-32).

Eso significa que, lo presentado alude a la importancia de que las TIC ayudan a modelos educativos más participativos, ampliando las coyunturas de investigación, comunicación y conocimiento. Por ende, la herramienta del Aprendizaje Colaborativo, servirá para conocer epistemológicamente el uso de los entornos virtuales, para hacer una buena inclusión en las asignaturas de informática en estudiantes universitarios del Instituto, Universitario Tecnológico Mario Briceño Iragorri, en el Estado Trujillo.

\subsection{Entornos Virtuales}

Las (TIC) como herramienta de instrucción, constituyen un recurso que viene a ayudar en cuanto a la manera de concebir conocimientos a grandes distancias y pequeños momentos de tiempo, de forma confiable. Desde esta perspectiva, señalan Aguilar y Vivas (2006), es importante resaltar que las TIC "son vistas como una herramienta que permite ir eliminando las brechas entre los quienes tienen con quienes no tienen acceso al conocimiento, viabilizando la inclusión social" (pág. 33). O sea, se debe minimizar el espacio que existe de quienes no puedan tener acceso libre al internet con los que sí lo poseen, porque en este momento la era de la tecnologización debe llegar a todo lugar para que la inmensa mayoría de sujetos puedan acceder a estos entornos virtuales y apoderarse de las bondades que ofrecen las TIC.

Al respecto, Delgado (2005), señala "se manifiestan en dos estratos: uno de naturaleza estructural «Infoestructura» y otro de naturaleza cultural «Infocultura»" (pág. 28). Interpretando los supuestos, estas representan elementos fundamentales en su avance que son importantes comprender, porque representan plataformas tecnológicas, proyectos elementales y de aplicación.

En este momento, con la aplicación de las (TIC), se han visto cambios 
en el espacio educativo. Estas ideas tecnológicas se corresponden con las idoneidades que debe tener el docente actual unido a las tendencias actuales referentes a la tecnologización. Tal como señalan, Gros y Silva (2003), "una formación mucho más centrada en el diseño de las situaciones y contexto de aprendizaje, en la mediación, tutorización y estrategias de aprendizaje" (pág. 56). De hecho, el currículo actual debe estar centrado donde el sujeto aprenda para desafiar nuevas situaciones que le ayuden a solucionar problemas cotidianos e inherentes al contexto que lo envuelve.

Igualmente, como lo menciona Martínez, (2003:78), citado en Villegas y Castillo, (2017), "las TIC ayudan al desarrollo de individuos, comunidades, regiones y países, además estas pueden mejorar los niveles educativos e impactar favorablemente en la calidad de vida para acceder a mejores oportunidades para los individuos, la sociedad y la educación" (pág. 201).

\subsubsection{Entornos Virtuales de Aprendizaje (EVA)}

La aplicabilidad tecnológica representa poder acceder a su manejo y aplicación, dado que el docente es quien debe emitir su valoración ante qué tipo de recursos pueden incorporar al proceso educativo, con modelos pedagógicos sobre los cuales basan su quehacer. Así, en los espacios educativos debe haber la representación adecuada debido a la accesibilidad generada cuando se desea interactuar con el recurso tecnológico. Al respecto, Zambrano, (2007), expresa: Estas tecnologías requieren de una gran responsabilidad en lo referente a la actualización de los medios... "debería de ponernos más alertas sobre cómo perciben nuestros alumnos la información que les aportamos a través de los medios tradicionales y buscar fórmulas capaces de poner en práctica procesos de aprendizaje que les haga mucho más atractiva la información" (pág. 3).

Con referencias a lo antes planteado, se manifiesta que, con los avances de la tecnología, el estudiante experimenta cambios cognitivos en 
virtud de enfrentar diferentes formas de aprender a aprehender, es decir, conocer las maneras de tener acceso y educarse a manejar los diferentes recursos tecnológicos para el aprendizaje. Asimismo, dichos constructos derivados del latín enseñanza cuyo significado es entendido como atrapar, conceptos empleados para hacer referencia a quienes se apropian del conocimiento, por tanto, las TIC, permitirá al estudiante conocer recursos tecnológicos, para ser incorporados en su práctica técnica futura.

Al respecto, Suárez, (2002), define los Entornos Virtuales de Aprendizaje (EVA), como: "un sistema de acción que basa su particularidad en una intención educativa y en una forma específica para lograrlo a través de recursos infovirtuales" (pág. 4). Plantea además, el mismo autor que "regula y transforma tecnológicamente la relación educativa de un modo definido otorgando a los sujetos formas de actuación externa para el aprendizaje". A su vez, con todos estos recursos el sujeto que aprende es capaz de modificar en su estructura interna lo aprendido sobre estrategias de pensamiento y aprendizaje. Ello implica, que EVA está referida a la organización del espacio, disposición y distribución de los recursos didácticos, manejo del tiempo e interacciones que se dan en el aula.

\subsubsection{Aproximaciones al Entorno Virtual del Futuro}

El entorno social que hoy se vive, está lejos de ser comparado con el de no muchos años atrás, la sociedad vive un desbalance cada vez más punteado debido a que se ven acentuadas la violencia y mucha prevalencia de antivalores que afectan la familia y la misma escuela y comunidad. Todo esto, ocasiona una descomposición social que impacta sin duda, el núcleo familiar. No obstante, la educación ha sido protagonista de este debacle contradictorio que ha adolecido a la sociedad actual.

De hecho, la escuela que hoy conocemos ha fracasado en su intento de educar y formar a hombres y mujeres adultos que en este momento 
conforman la sociedad que hoy vivimos, sin demostrar poseer la capacidad de lograr ambientes sanos, armónicos, que sean capaces de convivir en paz, libres de violencia. Por esto, se alude lo expresado por Ferreira y Labate, (2013a):

La decadencia de la sociedad actual urge un cambio de la educación hacia otros paradigmas de formación y acompañamiento en los aprendizajes de los niños y jóvenes que enfaticen el interior del ser humano y realmente generen individuos sanos física, mental y emocionalmente hablando, armónicos, pacíficos y competentes para desenvolverse en una sociedad productiva, tolerante, incluyente, sustentable y justa (pág. 9).

En otras palabras, la aproximación a una educación basada en entornos virtuales deberá hacer más hincapié en responder a hipótesis sobre la educación del futuro sobre a ¿Qué tipo de hombre deseamos para años venideros? Aquí, es de inferir que ha de tomarse en cuenta el perfil de egreso y el futuro de la educación, pareciera simple, pero en realidad es demasiado complejo.

Lo realmente interesante, es a juicio de Ferreira y Labate, (2013b), "tratar de contextualizar este espacio socioeducativo de la escuela dentro de un marco socio histórico en evolución dinámica y constante; es decir, los cambios de la humanidad y su relación con la tecnología y el conocimiento, a la luz de un lente ontológico diverso del ser humano individual" (pág. 10). Visto desde la perspectiva del autor, los nuevos avances tecnológicos se deben apoderar ontológicamente del ser humano porque el progreso como tal del campo de tecnología le hace a éste llegar a conocer y formarse en las dinámicas actuales para su incursión en entornos virtuales hasta ahora que pudieran ser desconocidos para él.

Visto así desde el ámbito educativo, debemos sacudir nuestros pensamientos y ocuparnos en que la escuela no puede ni debe estar al margen 
de la tecnología; se debe considerar las TIC como instrumentos colaterales indispensables para la formación del sujeto aprendiz. Por esto, las transformaciones que traerán los entornos virtuales ayudarán a comunicarse más rápidamente al individuo, donde la generación del futuro podrá visualizar la incertidumbre como algo normal adherida a su proceso de aprendizaje. Asimismo, las "comunidades virtuales", cada vez serán más agradables debido a su flexibilidad, diversidad e inclusión elementos propiciados en estas comunidades de aprendizaje.

\subsection{Comunidades Virtuales}

La realidad de hoy, en el mundo del conocimiento no escapa a ser diferente y esto es de observar en el contexto educativo, donde para aprender y aprehender la realidad ha habido cambios con el devenir de la época, y en la actualidad no se puede negar que el Internet ha instituido una red global donde se hace posible la conexión de las personas, teniendo la capacidad de comunicarse, generando un trabajo entre ellas, es decir en comunidad. Por tanto, Jonassen, Peck y Wilson (1999), citado en Salinas (2003a), indica con claridad que aparece una CV de Aprendizaje cuando:

Si una comunidad es una organización social de personas que comparten conocimiento, valores y metas, las clases como las conocemos no son comunidades ya que los estudiantes están desconectados o están compitiendo unos con otros. Las clases son comunidades sociales, pero su propósito no es aprender juntos o unos de otros...Las comunidades de aprendizaje surgen cuando los estudiantes comparten intereses comunes (pág. 5).

En otras palabras, cuando se hace referencia a comunidades virtuales el referente son comunidades de personas que conllevan valores y beneficios comunes, utilizando la comunicación mediante recursos telemáticos sean sincrónicos o asincrónicos, es decir estas comunidades no son inferiores a las 
presenciales. Así, aludiendo a Rheingold (1996), señala que cuando se produce un debate continuo en las CV éstas llegan a presentan las siguientes características (pág. 20):

- Están organizadas alrededor de afinidades y conducen a personas juntas que no necesariamente tienen que conocerse antes del encuentro en línea.

- Ellas exponen muchas personas y utilizan muchos medios.

- Se desarrollan desde el texto hasta la comunicación basada en gráficos.

- La comunicación se produce de forma más regular que en la comunicación cara a cara.

Estas características, las produce la interacción de grupos humanos que tienen puntos afines para logros de sus inquietudes, poseen semejanzas y su axiología personal la colocan para disentir saberes y opiniones que conllevan a intercambiar informaciones por vía Internet.

\subsection{Comunidad de Aprendizaje}

El Sistema educativo predominante centra su atención curricular más en el docente que en el ámbito familiar, llegando a observar que la inclusión de las familias se ve como una intromisión, debido a conflictos que puedan haberse suscitado por el grupo familiar debido a que su participación no fue la adecuada.

A todo esto, Salinas, (2003b:6), establece “...las comunidades profesionales, grupos de alumnos que siguen actividades académicas en un entorno virtual configurando comunidades virtuales, lo que requieren es disponer de una red de intercambio de información y el adecuado flujo de la información, colaboración, diversidad, compartir..." (pág. 60). Es decir, se hace necesario la formación permanente basada en que el docente se adapte a los nuevos recursos digitales en los ámbitos de la enseñanza-aprendizaje. 
Asimismo, Flecha y Puigvert, (2002), establecen:

Las Comunidades de Aprendizaje son un proyecto de transformación de centros educativos, dirigido a la superación del fracaso escolar y eliminación de conflictos. Este proyecto se distingue por una apuesta por el aprendizaje dialógico, mediante los grupos interactivos, donde el diálogo igualitario se convierte en un esfuerzo común para lograr la igualdad educativa de todos los alumnos (pág. 12).

En otras palabras, cuando sucede la transformación el cambio no sólo afecta al centro educativo, sino al entorno social más próximo, por esto se hace necesaria la participación y colaboración de las personas involucradas, para lograr las metas propuestas, para que el aprendizaje sea más significativo y haya más probabilidades de éxito.

\subsection{Teorías de Aprendizaje que Sustentan el Uso de los Entornos Virtuales.}

Entre las teorías de aprendizaje que sustentan el uso de los entornos virtuales en educación se encuentran: la pedagogía sociocrítica, el constructivismo y el conectivismo. Respecto, a la Pedagogía Social de corte Crítico-Social, se trata de hablar de una pedagogía más transformadora. Sobre todo, tal sello formativo debe apuntar a formar personas dispuestas a trabajar por el cambio profundo. Supone formar pedagogos preparados para diseñar y evaluar intervenciones de carácter innovador que de modo intencional modifique una realidad social, desde el diálogo y la participación.

Con Giroux, (1990), "estamos próximos a dibujar claramente el contenido de la Pedagogía Crítica o Sociocrítica" (pág. 145). En efecto, se sabe que la Pedagogía Crítica representa un cuerpo de saberes muy diverso y complejo cuyo propósito central viene dado por la necesidad de comprender y transformar la realidad socio escolar.

O lo que es lo mismo, lo original de la educación desde la mirada crítico- 
reflexiva, es visto desde el cuestionar y emancipar hacia la búsqueda en la construcción de nuevos sentidos axiológicos y diferentes formas de vida para el alcance de una sociedad justa y buena, aunque esto no se ha concretado. Lo demás, tales como contenidos, recursos didácticos, años de experiencia docente, el aumento de la jornada escolar, representan anexos de segundo orden y de naturaleza solamente instrumental. Dado que la acción transformadora solicita capacidad creativa, resulta fundamental examinar de otro modo este insumo, debidamente del modo que resulte oportuno a la racionalidad emancipadora que demanda una Pedagogía Sociocrítica.

En ese orden de ideas, el Constructivismo, según De La Torre, (2007), "implica un proceso de ordenación de componentes para construir un conocimiento" (pág. 49), es decir esto indica el involucramiento del ser humano integral, más no sólo su intelecto. De acuerdo, con Norman, (2008), el constructivismo se define como "un conjunto de teorías psicológicas que conciben los procesos cognitivos como construcciones eminentemente activas, resultado de la interacción del sujeto con el ambiente, los demás y consigo mismo" (pág. 138).

Conforme con lo anterior, el enfoque constructivista, es comunicación e interacción en busca de lograr resultados cognitivos, éticos, colectivos, además de soluciones a los problemas reales mediante la interacción teóricopráctica. Por lo demás la práctica del alumno es muy importante dentro del enfoque constructivista porque su finalidad: estriba en contribuir al desarrollo, es decir abrirse a experiencias superiores. Dewey, (1960), expone al respecto:

Algunas experiencias maleducan. Una experiencia maleduca cuando detiene o distorsiona el crecimiento de la experiencia posterior... Así como un hombre no vive ni muere para sí solo, tampoco una experiencia vive ni muere para sí sola. Por eso el problema central de una educación basada en la experiencia es seleccionar el tipo de experiencias actuales que sobrevivirán fructuosa y creativamente a la experiencia futura (págs. 25-28). 
Se puede inferir que la corriente del enfoque constructivista se ocupa del contenido de la enseñanza y del aprendizaje, privilegiando los conceptos y estructuras básicas de las ciencias, por encontrar según Bruner, (1980a), en "ellas un material de alta complejidad que brinda mejores oportunidades de desatar la capacidad intelectual del alumno y enseñarle como a un aprendiz de científico" (pág. 56).

Además, Bruner, (1980b), da por seguro que cualquier contenido científico puede ser interpretado desde la niñez si se les enseña bien y se le traduce a su lenguaje, "facilitando que los niños entiendan por sí mismos los conceptos básicos estructurales y los modos de investigar de cada ciencia, como en un aprendizaje por descubrimiento" (pág. 35). De esta manera, dicho aprendizaje está basado en el descubrimiento donde los estudiantes efectúan su aprendizaje a medida que experimentan y analizan la bibliografía disponible.

En consecuencia, a través de la teoría del conectivismo el conocimiento dentro de las instituciones educativas se puede formalizar mejor, al respecto Siemens, (2008), expone "conectivismo es una teoría del aprendizaje para la era digital basada en analizar limitaciones del conductismo, cognitivismo y constructivismo, para explicar el efecto que la tecnología ha tenido sobre la manera que actualmente vivimos, nos comunicamos y aprendemos" (pág. 43).

De hecho, el conectivismo es considerado como "la integración de los principios explorados por las teorías del caos, redes neuronales, complejidad y autoorganización Así, el aprendizaje es un asunto que acontece dentro de una amplia gama de entornos que no están precisamente controlados por el individuo y puede habitar fuera del ser humano, por ejemplo, dentro de una organización enfocándose en la conexión estudiada en conjuntos de información que permite desarrollar cada vez más nuestro estado actual de conocimiento. De manera concluyente, dada la importancia del conectivismo en el conocimiento se ha tomado como teoría del aprendizaje porque es capaz 
de observar las habilidades de aprendizaje y las tareas necesarias para que los estudiantes progresen en la era digital.

\section{Metodología}

Sin duda, en toda revisión documental se acude a un recorrido metodológico que representa el fundamento para el desarrollo del trabajo de exploración propiamente dicho. De acuerdo con Pardinas citado por Palella y Martins, (2004), "es el estudio crítico del método" (pág. 73).

Asimismo, la reflexión teórica es de tipo documental o bibliográfica, y llevan al investigador a utilizar textos, documentos de otra índole, elaborados previos al trabajo que se está investigando, tal como lo señala Sabino, (2005), "la investigación documental forma un procedimiento científico sistemático, de indagación, recolección, organización, interpretación y presentación de datos e información alrededor de una estrategia de análisis de documentos" (pág. 37).

Para llevar a cabo, esta revisión documental la primera fase consistió en permitir acercarse al área de estudio y profundizar el conocimiento sobre la situación a investigar, realizado a través de la recolección, organización y análisis de información comenzando por a estructurar los elementos que en principio sirvieron de partida al hecho investigativo, y que continuamente dan sustento y base a todo el proceso de exploración. Además, dicha revisión no se limitó al arqueo bibliográfico, los esfuerzos se rigieron a la observación de diferentes materiales didácticos en línea, revisión de experiencias en el área.

\section{Consideraciones Finales}

El uso de los entornos virtuales en el proceso de aprendizaje debe consistir en una innovación, creación y distinción del conocimiento debido al constante cambio social y tecnológico que aprecian las sociedades. Por esto, se presentaron los diversos enfoques o teorías de aprendizaje tales como la 
pedagogía sociocrítica, el constructivismo y el cognitivismo que reflejaron su aporte a la revisión mostrada, para dar a conocer al lector la importancia de ser tomadas en cuenta al momento de realizar trabajos basados en las tecnologías del conocimiento en la era digital.

Se reflejó, además lo significativo de las comunidades virtuales y del aprendizaje para ser ofrecidas como condiciones que permitan el uso de entornos virtuales como nuevas estrategias de aprendizaje en las aulas.

Las comunidades se forman a partir de los intereses de los propios usuarios, por lo que tal vez vivamos las vísperas de una revolución educativa. Donde cada individuo, sea capaz de unirse a las comunidades que sean más adecuadas, aporte sus conocimientos, experiencia y se nutra del de los demás, hasta lograr un nivel deseado, a favor de que cada sujeto podrá forjar su propio perfil profesional, recorriendo los caminos de formación que elija, según las insuficiencias de su entorno y sus propios intereses.

\section{Referencias}

Aguilar, S. \& Vivas, M. (2006). Enciclopedia de Informática. Bogotá: Editorial Norma.

Área, M. (2009a,b). Introducción a la Tecnología. Manual Electrónico. Universidad de La Laguna. Licencia: Creative Commons.

Bruner, J. (1980a,b). Investigaciones sobre el desarrollo cognitivo. Madrid: Pablo del Río.

Chiachio, G., Pievi, N., Echaverry, E., \& Gómez, V. (2009). La Educación en busca de Nuevos Sentidos. Buenos Aires. Jorge Baudino Ediciones. De La Torre, A. (2007). Aprender a aprender. Editorial: McGraw-Hill. Madrid. Delgado, M. (2005). Propuesta a docentes de educación media diversificada y profesional para la utilización de las TIC. Tesis Doctoral. Doctorado en Educación. Universidad Pedagógica Experimental Libertador. San Cristóbal estado Táchira, Venezuela. 
Dewey, J. (1960). Theory of the Moral Life. (Nueva York: Holt, Rinehart \& Winston, Inc.

Ferreira, A. \& Labate, L. (2013a,b). La educación de jóvenes en el futuro: Proyecciones 2030: desafíos y posibilidades. 1ra Edición diciembre 2013. Universidad Católica de Córdoba. Córdoba: Comunicarte.

Flecha, R. \& Puigvert, L. (2002). Las Comunidades de Aprendizaje. Una Apuesta por la Igualdad Educativa. REXE: Revista de Estudios y Experiencias en Educación. vol. 1, págs. 11-20.

Giroux, H. (1990). Los Profesores como Intelectuales. Hacia una Pedagogía Crítica del Aprendizaje. Barcelona: Paidós, pág. 178.

Gosden, C. (1994). Social Being and Time. Oxford: Blackwell.

Gros, B. \& Silva, J. (2003). La Formación del Profesorado como Docente en los Espacios Virtuales de Aprendizaje. Revista Iberoamericana de Educación. Recuperado de: http://rieoei.org/tec edu32.htm

Mestre, U. Fonseca, J. \& Valdez. P. (2007). Entornos virtuales de enseñanza aprendizaje. Monografía: Centro Universitario de Las Tunas, Ministerio de Educación Superior. Ciudad de Las Tunas: Editorial Universitaria.

Norman, S. (2008). Teorías del aprendizaje. Madrid: Editorial Anaya.

Palella S., \& Martins F. (2004). Metodología de la Investigación cuantitativa. Fondo Editorial de la Universidad Pedagógica Experimental Libertador, 204 páginas, Caracas, Venezuela.

Rheingold, H. (1996). La Comunidad Virtual. Una Sociedad sin fronteras. Barcelona: Gedisa Editorial. (Título original: The Virtual Community).

Sabino, C. (2005). "El proceso de Investigación". Caracas, Venezuela: Editorial: Panapo.

Salinas, J. (2003a,b). Comunidades Virtuales y Aprendizaje digital. Artículo presentado en el VI Congreso de Nuevas Tecnologías de la Información y de la comunicación para la educación. Venezuela: EDUTEC, 30 de junio a 4 de julio, Recuperado de: 
http://gte.uib.es/pape/gte/sites/gte.uib.es.pape.gte/files/Comunidades \%20Virtuales\%20y\%20Aprendizaje\%20Digital.pdf

Scagnoli, N. (2005a,b). Estrategias para Motivar el Aprendizaje Colaborativo en Cursos a Distancia. USA: College of Education University of Illinois at Urbana-Champaign.

Siemens, G. (2008) Learning and Knowing in Networks: Changing roles for Educators and Designers. Recuperado de:

https://www.academia.edu/2857165/Learning and knowing in networ ks Changing roles for educators and designers

Suárez, (2002). Entornos Virtuales de Aprendizaje como Instrumento de Mediación. España: Ediciones Universidad de Salamanca.

Villegas, M., \& Castillo, R. (2017). Uso de las Tic en la Formación Permanente de los Docentes Asesores en la Universidad. Revista Scientific, 2(6), 196-216. Recuperado de:

https://doi.org/10.29394/scientific.issn.2542-2987.2017.2.6.10.196-216 Zambrano, D. (2007). Revisión a los Recursos Educativos Abiertos. Madrid: Editorial Popular. 


\section{Elide del Rosario Castellanos Santiago}

\section{e-mail: elidecastellanos255@hotmail.com}

Lugar de nacimiento, Valera estado Trujillo, Venezuela.

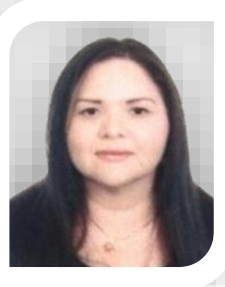

Curso estudio de: Doctorado en Educación. en Universidad Nacional Experimental Rafael María Baralt en Cabimas estado Zulia, Venezuela. Licenciatura en Educación Integral en La U.N.E.R.M.B. Titulo Obtenido: Licenciada en Educación Integral. Fecha: 18 de marzo de 2005. Maestría en Docencia para Educación Superior en La U.N.E.R.M.B. Titulo Obtenido: Magister Scientiarum en Docencia para Educación Superior. Fecha: 02 de diciembre de 2010.

En la actualidad, se desempeña como docente en el Ministerio del Poder Popular para la Educación. N.E.R. 233 desde 16 de septiembre 2005 hasta la presente y en la Misión Sucre Aldea Universitaria "Cruz Carrillo" Como: Profesora Asesora del Programa Nacional de Gestión Social, en Sabana de Mendoza desde septiembre del 2008 hasta el presente año 2017. 


\section{Javier José Castro Capitillo}

e-mail: jccapitillo@hotmail.com

Nacido en Cabimas estado Zulia, Venezuela. Cursó

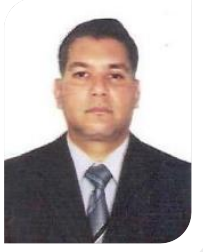
estudios en: Universidad Nacional Experimental "Rafael María Baralt" Título obtenido: Licenciado en Administración: Mención Gerencia Industrial. Fecha: octubre 1999, Cabimas estado Zulia, Venezuela.

Universidad Nacional Experimental "Rafael María Baralt" Título obtenido: Magister Scientiarum en Gerencia de Recursos Humanos. Fecha: mayo 2010, Maracaibo estado Zulia, Venezuela.

Universidad Nacional Experimental "Rafael María Baralt" En curso: Doctorado en Educación. Fecha: febrero 2017, Cabimas estado Zulia, Venezuela.

Universidad Nacional Experimental "Rafael María Baralt" Título obtenido: "Formación Docente para Egresados". (Componente Docente). Fecha: septiembre 2006-2007, Cabimas estado Zulia, Venezuela.

Actualmente me desempeño en la Universidad Nacional Experimental "Rafael María Baralt, Sede Mene Grande, Mene Grande Municipio Baralt, estado Zulia, Venezuela. Cargo: Docente de las materias: Gerencia de Mercado, Taller de Acción Profesional I y II, Matemáticas II, Estrategias Gerencial. Finanzas de la Empresa. (Programa de Administración). Fundamentos Matemáticos. Aritmética I (Programa de Educación) Períodos: abril 2005, Período actual (2017).

El contenido de este manuscrito se difunde bajo una Licencia de Creative Commons ReconocimientoNoComercial-Compartirlgual 4.0 Internacional 